\title{
Some remarks on symmetric correspondences
}

\author{
H. Lange \\ Mathematisches Institut \\ Universitat Erlangen-Nurnberg \\ Bismarckstr. $1 \frac{1}{2}$ \\ D-91054 Erlangen (Germany) \\ E. Sernesi \\ Dipartimento di Matematica \\ Università Roma Tre \\ Largo S.L. Murialdo 1 \\ 00146 Roma (italy)
}

\section{Introduction}

A $\left(d_{1}, d_{2}\right)$-correspondence on a projective connected nonsingular curve $C$ can be defined as a curve $D \subset C \times C$ of type $\left(d_{1}, d_{2}\right)$. The $i$-th projection restricts to a morphism of degree $d_{i}$

$$
p_{i}: D \rightarrow C
$$

for $i=1,2$. These morphisms induce a correspondence in the usual sense, associating to a point $x \in C$ the divisor $p_{2}\left(p_{1}^{-1}(x)\right)$ of degree $d_{1}$. On any curve $C$ there exist plenty of correspondences, but if the curve is general they are all correspondences with valency: namely there is an integer $N$ such that the linear equivalence class of the divisor

$$
p_{2}\left(p_{1}^{-1}(x)\right)+N x
$$

is independent of $x$. It is more difficult to find correspondences without valency. 
A $(d, d)$-correspondence is called symmetric if it is mapped to itself by the involution on $C \times C$ which interchanges the factors. Hence for a symmetric correspondence $D$, the involution on $C \times C$ induces an involution on the curve $D$. If we assume that the correspondence is without fixed points, i.e. it does not meet the diagonal $\Delta \subset C \times C$, the involution on $D$ is fixed-point free. This is the specific case that we will study in this paper. The interest of such correspondences comes from their relation with the theory of Prym-Tyurin varieties. We refer the reader to [1] for details on this theory and on how it is related with correspondences.

We consider a few geometrical configurations arising from a nonsingular symmetric fixed-point free $(d, d)$-correspondence and we study their deformation theory. This gives informations on the deformation theory of PrymTyurin varieties, a subject on which very little is known.

We are able to compute several invariants and to test them on a few known examples.

\section{The second symmetric product}

Let $C$ be a complex projective nonsingular connected curve of genus $g \geq 2$. Denote by $C^{n}$ and $C_{n}$ the $n$-th cartesian, resp. symmetric, product of $C$, for any $n>0$. We will be interested in the case $n=2$.

In this section we want to compute the cohomology of $T_{C_{2}}$, the tangent bundle of $C_{2}$. For this purpose consider the universal divisor

$$
\Delta_{2}=\{(x, E): E-x>0\} \subset C \times C_{2}
$$

and let

$$
\begin{aligned}
& C \times C_{2} \\
& \begin{array}{ll}
\downarrow p & \searrow q \\
C & C_{2}
\end{array}
\end{aligned}
$$

be the projections. We have the well known formula

$$
T_{C_{2}}=q_{*}\left[\mathcal{O}_{\Delta_{2}}\left(\Delta_{2}\right)\right]
$$

(see [4]). We have an obvious isomorphism

$$
\begin{array}{ccc}
C \times C & \stackrel{\epsilon}{\longrightarrow} & \Delta_{2} \\
(x, y) & \mapsto & (x, x+y)
\end{array}
$$


and we can identify the natural map $\sigma: C \times C \rightarrow C_{2}$ with the composition

$$
C \times C \stackrel{\epsilon}{\longrightarrow} \Delta_{2} \stackrel{q}{\longrightarrow} C_{2}
$$

Moreover the composition $p \epsilon: C \times C \rightarrow C$ gets identified with the first projection which we will keep calling $p$. Now, using the identity

$$
\epsilon^{*} \mathcal{O}_{\Delta_{2}}\left(\Delta_{2}\right)=p^{*} \omega_{C}^{-1} \otimes \mathcal{O}(\Delta)
$$

(see [4], lemma 1.1. Warning: $\Delta$ is the diagonal in $C \times C$ while $\Delta_{2}$ is the diagonal in $C \times C_{2}$ ) we can reformulate (1) as follows:

$$
T_{C_{2}}=\sigma_{*}\left[p^{*} \omega_{C}^{-1}(\Delta)\right]
$$

Since $\sigma$ is finite we have canonical identifications:

$$
H^{i}\left(T_{C_{2}}\right)=H^{i}\left(p^{*} \omega_{C}^{-1}(\Delta)\right), \quad i=0,1,2
$$

Lemma 1.1

$$
\begin{aligned}
& h^{0}\left(p^{*} \omega_{C}^{-1}\right)=0 \\
& h^{1}\left(p^{*} \omega_{C}^{-1}\right)=3 g-3 \\
& h^{2}\left(p^{*} \omega_{C}^{-1}\right)=g(3 g-3)
\end{aligned}
$$

and therefore

$$
\chi\left(p^{*} \omega_{C}^{-1}\right)=3(g-1)^{2}
$$

Proof. Follows easily from the Kunneth formula or Leray spectral sequence.

Lemma 1.2 If $g \geq 3$ then

$$
h^{0}\left(R^{1} p_{*} \mathcal{O}(\Delta) \otimes \omega_{C}^{-1}\right)=0
$$

and

$$
h^{1}\left(R^{1} p_{*} \mathcal{O}(\Delta) \otimes \omega_{C}^{-1}\right)=3 g^{2}-8 g+5
$$

If $g=2$ then $R^{1} p_{*} \mathcal{O}(\Delta) \cong \omega_{C}$. 
Proof. Assume $g \geq 3$. By pushing down by $p$ the exact sequence

$$
0 \rightarrow \mathcal{O} \rightarrow \mathcal{O}(\Delta) \rightarrow \omega_{\Delta}^{-1} \rightarrow 0
$$

we obtain the exact sequence on $C$ :

$$
0 \rightarrow \mathcal{O}_{C} \rightarrow p_{*} \mathcal{O}(\Delta) \rightarrow \omega_{C}^{-1} \rightarrow R^{1} p_{*} \mathcal{O}_{C} \rightarrow R^{1} p_{*} \mathcal{O}(\Delta) \rightarrow 0
$$

We have $R^{1} p_{*} \mathcal{O}_{C}=H^{1}\left(\mathcal{O}_{C}\right) \otimes \mathcal{O}_{C}$ and by rank reasons $O_{C} \cong p_{*} \mathcal{O}(\Delta)$. We are left with the exact sequence

$$
0 \rightarrow \omega_{C}^{-1} \rightarrow H^{1}\left(\mathcal{O}_{C}\right) \otimes \mathcal{O}_{C} \rightarrow R^{1} p_{*} \mathcal{O}(\Delta) \rightarrow 0
$$

which is a twist of the Euler sequence of $\mathbb{P}^{g-1}$ restricted to $C$ via the canonical morphism $C \rightarrow \mathbb{P}^{g-1}$; in particular

$$
R^{1} p_{*} \mathcal{O}(\Delta) \cong T_{\mathbb{P}^{g-1} \mid C} \otimes \omega_{C}^{-1}
$$

Let $\mathbf{a}=x_{1}+\cdots+x_{g-2}$ be a general effective divisor of degree $g-2$ on $C$. Then there is an exact sequence ( see [3], (2.3): the proof is valid in the hyperelliptic case as well, see [6], , Lemma 1.4.1)

$$
0 \rightarrow \omega_{C}^{-1}(\mathbf{a}) \rightarrow R^{1} p_{*} \mathcal{O}(\Delta)^{\vee} \rightarrow \bigoplus \mathcal{O}_{C}\left(-x_{i}\right) \rightarrow 0
$$

Dualizing and twisting by $\omega_{C}^{-1}$ we obtain:

$$
0 \rightarrow \bigoplus \omega_{C}^{-1}\left(x_{i}\right) \rightarrow R^{1} p_{*} \mathcal{O}(\Delta) \otimes \omega_{C}^{-1} \rightarrow \mathcal{O}(-\mathbf{a}) \rightarrow 0
$$

which implies $h^{0}\left(R^{1} p_{*} \mathcal{O}(\Delta) \otimes \omega_{C}^{-1}\right)=0$. The other estimate follows also from this sequence.

The case $g=2$ follows from the exact sequence (5).

The following result is well known (see [2]):

Proposition 1.3 If $g \geq 3$ then

$$
\begin{aligned}
h^{0}\left(T_{C_{2}}\right) & =0 \\
h^{1}\left(T_{C_{2}}\right) & =3 g-3 \\
h^{2}\left(T_{C_{2}}\right) & =3 g^{2}-8 g+5
\end{aligned}
$$

and therefore

$$
\chi\left(T_{C_{2}}\right)=3 g^{2}-11 g+8
$$

If $g=2$ then $h^{0}\left(T_{C_{2}}\right)=0, h^{1}\left(T_{C_{2}}\right)=4$ and $h^{2}\left(T_{C_{2}}\right)=2$. 
Proof. The equality $h^{0}\left(T_{C_{2}}\right)=0$ is an immediate consequence of (3) for all $g \geq 2$.

Assume $g \geq 3$. By the Leray spectral sequence for $p: C \times C \rightarrow C$ and equation (3) we have an exact sequence

$$
0 \rightarrow H^{1}\left(\omega_{C}^{-1} \otimes p_{*} \mathcal{O}(\Delta)\right) \rightarrow H^{1}\left(T_{C_{2}}\right) \rightarrow H^{0}\left(\omega_{C}^{-1} \otimes R^{1} p_{*} \mathcal{O}(\Delta)\right) \rightarrow 0
$$

The exact sequence (4) implies that $p_{*} \mathcal{O}(\Delta)=\mathcal{O}_{C}$ so that we have

$$
H^{1}\left(T_{C_{2}}\right)=H^{1}\left(\omega_{C}^{-1}\right) \cong \mathbf{C}^{3 g-3}
$$

because $H^{0}\left(\omega_{C}^{-1} \otimes R^{1} p_{*} \mathcal{O}(\Delta)\right)=0$ by Lemma 1.2. Thethe Leray spectral sequence for $p: C \times C \rightarrow C$ and equation (3) again we have

$$
H^{2}\left(T_{C_{2}}\right) \cong H^{1}\left(R^{1} p_{*} \mathcal{O}(\Delta) \otimes \omega_{C}^{-1}\right)
$$

and again the conclusion follows from Lemma 1.2.

If $g=2$ the exact sequence (6) becomes:

$$
0 \rightarrow H^{1}\left(\omega_{C}^{-1}\right) \rightarrow H^{1}\left(T_{C_{2}}\right) \rightarrow H^{0}\left(\mathcal{O}_{C}\right) \rightarrow 0
$$

by Lemma 1.2. Therefore $h^{1}\left(T_{C_{2}}\right)=4$. Similarly $(7)$ in this case gives

$$
H^{2}\left(T_{C_{2}}\right) \cong H^{1}\left(\mathcal{O}_{C}\right) \cong \mathbf{C}^{2}
$$

\section{The set-up}

Let $D \subset C \times C$ be a symmetric irreducible and nonsingular curve of type $(d, d)$. Assume that $D \cdot \Delta=0$, where $\Delta \subset C \times C$ is the diagonal. In particular $D$ is a symmetric fixed-point free $(d, d)$-correspondence on $C$.

We have the following situation:

$$
\begin{array}{clll}
C \times C & \stackrel{\sigma}{\longrightarrow} & C_{2} \\
\cup & & \cup \\
D & \rightarrow & \bar{D} \\
& \downarrow p \\
C & & \\
& &
\end{array}
$$


Here $p$ is induced by the projection, $\sigma$ is the canonical quotient map, $\bar{D}:=$ $\sigma(D)$ is irreducible and nonsingular and the lower horizontal map is an etale double cover.

Let $g(D)$ and $\bar{g}$ be the genera of $D$ and of $\bar{D}$ respectively. By the adjunction formula on $C \times C$ we have:

$$
g(D)-1=2 d(g-1)+\frac{D^{2}}{2}=2 d(g-1)+\bar{D}^{2}
$$

On the other hand by Hurwitz formula applied to $p$ we have

$$
2(g(D)-1)=2 d(g-1)+|R|
$$

where $|R|$ is the degree of the ramification divisor $R$ of $p: D \rightarrow C$. By comparing the two formulas we therefore get:

$$
\bar{D}^{2}=-g(D)+1+|R|
$$

But since $g(D)-1=2 \bar{g}-2$ we also have:

$$
\bar{D}^{2}=2 \bar{g}-2-2 d(g-1)=2-2 \bar{g}+|R|
$$

From these expressions we get the following Riemann-Roch formula for the normal sheaf $N_{\bar{D}}$ of $\bar{D} \subset C_{2}$ :

$$
\chi\left(N_{\bar{D}}\right)=3-3 \bar{g}+|R|
$$

equivalently:

$$
\chi\left(N_{\bar{D}}\right)=\bar{g}-1-2 d(g-1)
$$

Consider the exact sequence on $C \times C$ :

$$
0 \rightarrow p^{*} \omega_{C}^{-1}(\Delta-D) \rightarrow p^{*} \omega_{C}^{-1}(\Delta) \rightarrow p^{*} \omega_{C}^{-1} \otimes \mathcal{O}_{D} \rightarrow 0
$$

(recall that $D \cdot \Delta=0$ ). Taking $\sigma_{*}$ we obtain the following exact sequence on $C_{2}$ :

$$
0 \rightarrow \sigma_{*}\left[p^{*} \omega_{C}^{-1}(\Delta-D)\right] \rightarrow T_{C_{2}} \rightarrow \sigma_{*}\left[p^{*} \omega_{C}^{-1} \otimes \mathcal{O}_{D}\right] \rightarrow 0
$$

Now, since the sheaf on the right is locally free of rank two and it is supported on $\bar{D}$, the last map factors as the restriction $T_{C_{2}} \rightarrow T_{C_{2} \mid \bar{D}}$ composed with a surjection of locally free rank two sheaves

$$
T_{C_{2} \mid \bar{D}} \rightarrow \sigma_{*}\left[p^{*} \omega_{C}^{-1} \otimes \mathcal{O}_{D}\right]
$$


which must therefore be an isomorphism. It also follows that

$$
\sigma_{*}\left[p^{*} \omega_{C}^{-1}(\Delta-D)\right] \cong T_{C_{2}}(-\bar{D})
$$

Using the fact that $\sigma$ is finite we can state the following:

Lemma 2.1 For all $i$ we have:

$$
\begin{aligned}
& H^{i}\left(T_{C_{2}}(-\bar{D})\right) \cong H^{i}\left(p^{*} \omega_{C}^{-1}(\Delta-D)\right) \\
& H^{i}\left(T_{C_{2} \mid \bar{D}}\right) \cong H^{i}\left(p^{*} \omega_{C}^{-1} \otimes \mathcal{O}_{D}\right)
\end{aligned}
$$

and the cohomology sequence of (13) is isomorphic to the cohomology sequence of

$$
0 \rightarrow T_{C_{2}}(-\bar{D}) \rightarrow T_{C_{2}} \rightarrow T_{C_{2} \mid \bar{D}} \rightarrow 0
$$

We will need the following exact and commutative diagram:

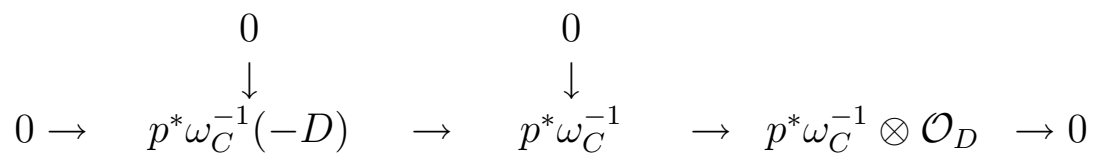

$$
\begin{aligned}
& 0 \rightarrow p^{*} \omega_{C}^{-1}(\Delta-D) \rightarrow p^{*} \omega_{C}^{-1}(\Delta) \rightarrow p^{*} \omega_{C}^{-1} \otimes \mathcal{O}_{D} \rightarrow 0 \\
& \begin{array}{ccc} 
& \downarrow & \\
\omega_{\Delta}^{-2} & = & \omega_{\Delta}^{-2} \\
\downarrow & & \downarrow \\
0 & & 0
\end{array}
\end{aligned}
$$

Lemma 2.2 If $\bar{g} \geq 2$ then

$$
\begin{aligned}
& h^{i}\left(p^{*} \omega_{C}^{-1}(-D)\right)=0, \quad i=0,1 \\
& h^{2}\left(p^{*} \omega_{C}^{-1}(-D)\right)=(g-1)[3(g-1)+4 d]+\bar{D}^{2}
\end{aligned}
$$

Proof. Set $E=p^{*} \omega_{C}(D)$. Then

$$
\begin{aligned}
E \cdot D & =p^{*} \omega_{C} \cdot D+2 \bar{D}^{2}= \\
& =2 d(g-1)+2[2 \bar{g}-2-2 d(g-1)]=4(\bar{g}-1)-2 d(g-1) \\
& =\bar{D}^{2}+2 \bar{g}-2=|R| \geq 0
\end{aligned}
$$


Moreover

$$
\begin{aligned}
& E^{2}=D^{2}+2 d(2 g-2)=2 \bar{D}^{2}+4 d(g-1)= \\
& =2[2 \bar{g}-2-2 d(g-1)]+4 d(g-1) \quad=4(\bar{g}-1)>0
\end{aligned}
$$

Therefore, since $E$ is effective, it is big and nef. Therefore the first part follows from the Kawamata-Viehweg vanishing theorem.

Since $\operatorname{deg}\left(p^{*} \omega_{C}^{-1} \otimes \mathcal{O}_{D}\right)=2 d(1-g)$, we have

$$
\chi\left(p^{*} \omega_{C}^{-1} \otimes \mathcal{O}_{D}\right)=2 d(1-g)+1-g(D)=4 d(1-g)-\bar{D}^{2}
$$

Using the exact sequence

$$
0 \rightarrow p^{*} \omega_{C}^{-1}(-D) \rightarrow p^{*} \omega_{C}^{-1} \rightarrow p^{*} \omega_{C}^{-1} \otimes \mathcal{O}_{D} \rightarrow 0
$$

and recalling Lemma 1.1 we obtain:

$$
\begin{array}{ll}
h^{2}\left(p^{*} \omega_{C}^{-1}(-D)\right)=\chi\left(p^{*} \omega_{C}^{-1}(-D)\right) & = \\
=\chi\left(p^{*} \omega_{C}^{-1}\right)-\chi\left(p^{*} \omega_{C}^{-1} \otimes \mathcal{O}_{D}\right) & =(g-1)[3(g-1)+4 d]+\bar{D}^{2}
\end{array}
$$

Proposition 2.3 If $\bar{g} \geq 2$ then

$$
\begin{gathered}
h^{0}\left(T_{C_{2}}(-\bar{D})\right)=0 \\
h^{1}\left(T_{C_{2}}(-\bar{D})\right)=0 \\
\chi\left(T_{C_{2}}(-\bar{D})\right)=h^{2}\left(T_{C_{2}}(-\bar{D})\right)=(g-1)[3(g-1)+4 d-5]+\bar{D}^{2}
\end{gathered}
$$

Proof. The equality $h^{0}\left(T_{C_{2}}(-\bar{D})\right)=0$ is clear. By Lemma 2.1 it suffices to show that $H^{1}\left(p^{*} \omega_{C}^{-1}(\Delta-D)\right)=0$ in order to prove the second equality. We will use diagram $(16)$. Note that $H^{0}(\mathcal{F})=0$ for all sheaves $\mathcal{F}$ in the diagram. Moreover, since $H^{1}\left(p^{*} \omega_{C}^{-1}(-D)\right)=0$ (Lemma 2.2), the first column of (16) shows that it suffices to prove that

$$
H^{1}\left(\omega_{\Delta}^{-2}\right) \rightarrow H^{2}\left(p^{*} \omega_{C}^{-1}(-D)\right)
$$

is injective. This amounts to show that the coboundary map

$$
H^{1}\left(\omega_{\Delta}^{-2}\right) \rightarrow H^{2}\left(p^{*} \omega_{C}^{-1}\right)
$$


coming from the second column of (16) is injective. But the cohomology sequence of the second column of (16) is

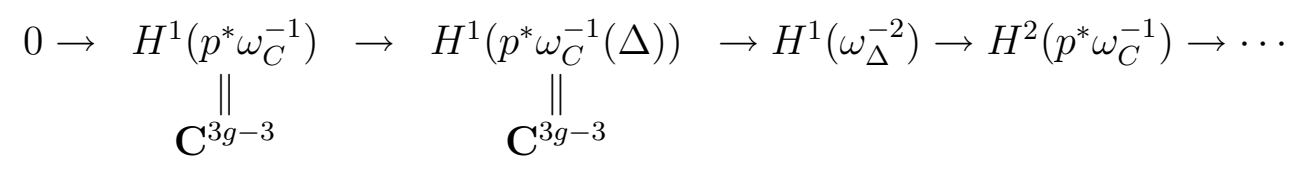

where we used Lemma 1.1, equation (3) and Proposition 1.3; so this proves that $h^{1}\left(T_{C_{2}}(-\bar{D})\right)=0$. The identity for $h^{2}\left(T_{C_{2}}(-\bar{D})\right)$ follows from Lemma 2.1 and the first part and from the cohomology sequence of the first column of diagram (16).

We now prove a result which is useful for the computation of the cohomology of $T_{C_{2} \mid \bar{D}}$.

Proposition 2.4 We have an identity

$$
T_{C_{2} \mid \bar{D}}=\omega_{\bar{D}}^{-1} \otimes \sigma_{*} \mathcal{O}(R)
$$

and an exact sequence:

$$
0 \rightarrow \omega_{\bar{D}}^{-1} \oplus \omega_{\bar{D}}^{-1} \eta \rightarrow T_{C_{2} \mid \bar{D}} \rightarrow \mathbf{t}^{\prime} \rightarrow 0
$$

where $\mathbf{t}^{\prime}$ is a torsion sheaf with $h^{0}\left(\mathbf{t}^{\prime}\right)=|R|$.

Proof. We have the identifications

$$
p^{*} \omega_{C}^{-1} \otimes \mathcal{O}_{D}=\omega_{D}^{-1}(R)=\sigma^{*} \omega_{\bar{D}}^{-1}(R)
$$

The second one being because $D \rightarrow \bar{D}$ is unramified. Then by applying the projection formula we have

$$
\sigma_{*}\left[p^{*} \omega_{C}^{-1} \otimes \mathcal{O}_{D}\right]=\omega_{\bar{D}}^{-1} \otimes \sigma_{*} \mathcal{O}(R)
$$

and therefore, after recalling the isomorphism (15), we have the identity (17).

Let $\eta \in \operatorname{Pic}_{2}(\bar{D})$ be the 2-torsion point defining the double cover $D \rightarrow \bar{D}$. Then we have $\sigma_{*} \mathcal{O}_{D}=\mathcal{O}_{\bar{D}} \oplus \eta$ and the exact sequence on $\bar{D}$

$$
0 \rightarrow \mathcal{O}_{\bar{D}} \oplus \eta \rightarrow \sigma_{*} \mathcal{O}(R) \rightarrow \mathbf{t} \rightarrow 0
$$

where $\mathbf{t}$ is a torsion sheaf with $h^{0}(\mathbf{t})=|R|$ obtained by pushing down the obvious sequence on $D$ :

$$
0 \rightarrow \mathcal{O}_{D} \rightarrow \mathcal{O}_{D}(R) \rightarrow \mathcal{O}_{R}(R) \rightarrow 0
$$

Tensoring with $\omega_{\bar{D}}^{-1}$ and using (17) we obtain (18). 
Example 2.5 (The classical Prym varieties) Let $C$ have a fixed-point free involution $\iota$ and let $D \subset C \times C$ be the graph of $\iota$. In this case $d=1$, $R=0, D=C$, and $g=g(D)=2 \bar{g}-1$. Assume $\bar{g} \geq 2$, i.e. $g \geq 3$. Using (10) we get $\operatorname{deg}\left(N_{\bar{D}}\right)=-g+1<0$ and, by (11):

$$
h^{0}\left(N_{\bar{D}}\right)=0, \quad h^{1}\left(N_{\bar{D}}\right)=-\chi\left(N_{\bar{D}}\right)=3 \bar{g}-3
$$

Moreover by (18) we have

$$
h^{1}\left(T_{C_{2} \mid \bar{D}}\right)=h^{0}\left(\omega_{\bar{D}}^{\otimes 2}\right)+h^{0}\left(\eta \omega_{\bar{D}}^{\otimes 2}\right)=6 \bar{g}-6
$$

On the other hand

$$
h^{1}\left(T_{\bar{D}}\right)=3 \bar{g}-3
$$

and, by Proposition 1.3:

$$
h^{1}\left(T_{C_{2}}\right)=h^{1}\left(T_{C}\right)=3(2 \bar{g}-1)-3=6 \bar{g}-6
$$

Therefore, since $h^{1}\left(T_{C_{2}}(-\bar{D})\right)=0$ (Proposition 2.3), we see that the restriction map

$$
H^{1}\left(T_{C_{2}}\right) \rightarrow H^{1}\left(T_{C_{2} \mid \bar{D}}\right)
$$

is an isomorphism, and that:

$$
H^{1}\left(T_{C_{2}}\right) \cong H^{1}\left(T_{C_{2} \mid \bar{D}}\right) \cong H^{1}\left(T_{\bar{D}}\right) \oplus H^{1}\left(N_{\bar{D}}\right)
$$

\section{Deformations of the correspondence}

Let the notation be as in the last section. Let

$$
T_{C_{2}}\langle\bar{D}\rangle \subset T_{C_{2}}
$$

be the inverse image of $T_{\bar{D}} \subset T_{C_{2} \mid \bar{D}}$ under the natural restriction homomorphism:

$$
T_{C_{2}} \rightarrow T_{C_{2} \mid \bar{D}}
$$

The deformation theory of the pair $\left(\bar{D}, C_{2}\right)$ is controlled by the sheaf $T_{C_{2}}\langle\bar{D}\rangle$. Precisely the tangent and the obstruction space of the deformation functor of the pair are $H^{1}\left(C_{2}, T_{C_{2}}\langle\bar{D}\rangle\right)$ and $H^{2}\left(C_{2}, T_{C_{2}}\langle\bar{D}\rangle\right)$ respectively (see [7], §3.4.4). 
For the computation of these two vector spaces we consider the following exact sequences on $C_{2}$ :

$$
0 \rightarrow T_{C_{2}}(-\bar{D}) \rightarrow T_{C_{2}}\langle\bar{D}\rangle \rightarrow T_{\bar{D}} \rightarrow 0
$$

and

$$
0 \rightarrow T_{C_{2}}\langle\bar{D}\rangle \rightarrow T_{C_{2}} \rightarrow N_{\bar{D}} \rightarrow 0
$$

which fit into the commutative diagram with exact rows and columns:

$$
\begin{aligned}
& \begin{array}{ll}
0 & 0
\end{array} \\
& \stackrel{\downarrow}{\downarrow} T_{C_{2}}(-\bar{D})=T_{C_{2}}(-\bar{D}) \\
& 0 \rightarrow T_{C_{2}}\langle\bar{D}\rangle \quad \rightarrow \quad T_{C_{2}} \quad \rightarrow \quad N_{\bar{D}} \rightarrow 0
\end{aligned}
$$

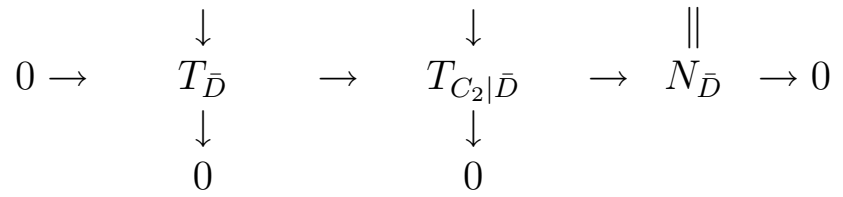

As a consequence of Proposition 1.3 we obtain

$$
h^{0}\left(T_{C_{2}}\langle\bar{D}\rangle\right)=0
$$

In view of Proposition 2.3 if $\bar{g} \geq 2$ then the cohomology of this diagram is the following:

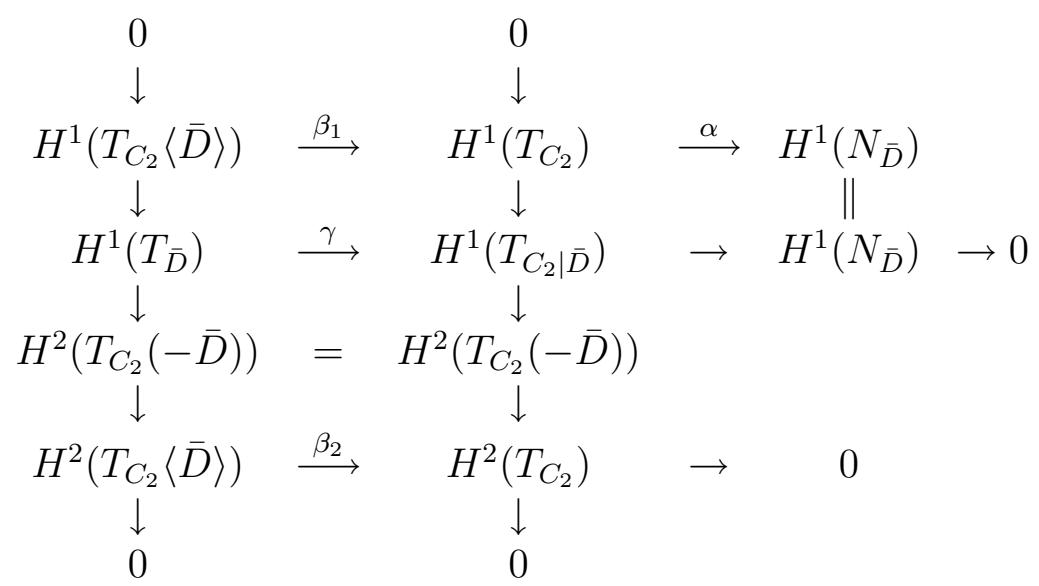


Note that

$$
\begin{aligned}
& \operatorname{ker}\left(\beta_{2}\right)=\operatorname{coker}(\alpha) \\
& \operatorname{ker}\left(\beta_{1}\right)=H^{0}\left(N_{\bar{D}}\right) \quad=\operatorname{ker}(\gamma)
\end{aligned}
$$

In general we have

$$
h^{1}\left(T_{C_{2} \mid \bar{D}}\right) \neq 0 \neq h^{1}\left(N_{\bar{D}}\right)
$$

so that $\bar{D}$ is neither stable nor costable in $C_{2}$ (see [7], $§ 3.4 .5$, for the definition of stability and costability).

\section{Lemma 3.1}

$$
\chi\left(T_{C_{2}}\langle\bar{D}\rangle\right)=1-\bar{g}+(g-1)(3 g+2 d-8)
$$

Proof. From the exact sequence (20) we deduce the following identity:

$$
\chi\left(T_{C_{2}}\langle\bar{D}\rangle\right)=\chi\left(T_{C_{2}}(-\bar{D})\right)+\chi\left(T_{\bar{D}}\right)
$$

Using Proposition 2.3 and (10) we obtain:

$\chi\left(T_{C_{2}}\langle\bar{D}\rangle\right)=\bar{D}^{2}+(g-1)(3 g+4 d-8)+3-3 \bar{g}=1-\bar{g}+(g-1)(3 g+2 d-8)$

Remarks 3.2 If we denote by $\mu\left(\bar{D}, C_{2}\right)$ the number of moduli of the pair $\left(\bar{D}, C_{2}\right)$ (see [7]) then we have:

$$
-\chi\left(T_{C_{2}}\langle\bar{D}\rangle\right) \leq \mu\left(\bar{D}, C_{2}\right) \leq h^{1}\left(T_{C_{2}}\langle\bar{D}\rangle\right)
$$

The second inequality is an equality if and only if the pair $\left(\bar{D}, C_{2}\right)$ is unobstructed. Since $\chi\left(T_{C_{2}}\langle\bar{D}\rangle\right)$ tends to be positive, the lower bound is negative in general and therefore it does not give any useful information about the deformations of the pair.

Remarks 3.3 The sheaf $T_{C_{2}}(-\bar{D})$ controls the deformations of $\left(\bar{D}, C_{2}\right)$ which induce a trivial deformation of $\bar{D}$. The fact that $H^{1}\left(T_{C_{2}}(-\bar{D})\right)=0$ (Proposition 2.3) means that there are no such infinitesimal deformations. This can be explained geometrically as follows.

To deform $\left(\bar{D}, C_{2}\right)$ without deforming $\bar{D}$ is the same as deforming $\left(D, C^{2}\right)$ without deforming $D$ (because $D$ is etale over $\bar{D}$ ). This means to deform $C$ without deforming its cover $D$ and this is not possible because it would contradict the theorem of de Franchis for $D$. 


\section{Examples}

We consider a few examples and we apply the previous calculations.

1. The classical Prym varieties - This is the example considered at the end of $\S 2$. By comparing the expressions given by Propositions 1.3 and 2.3 we get

$$
h^{2}\left(T_{C_{2}}(-\bar{D})\right)=h^{2}\left(T_{C_{2}}\right)
$$

and from this it immediately follows that

$$
H^{1}\left(T_{C_{2}}\langle\bar{D}\rangle\right)=H^{1}\left(T_{\bar{D}}\right)
$$

In particular the space of first order deformations of the pair $\left(\bar{D}, C_{2}\right)$, i.e. those which preserve the involution $\iota$ on $C$, has dimension $3 \bar{g}-3=3 \operatorname{dim}(P)$, where $P$ is the Prym variety of the pair $(C, \iota)$, a very well known fact. The map $\beta_{2}$ of diagram (24) is bijective because $\alpha$ is surjective.

2. Let $f: X \rightarrow X^{\prime}$ be an unramified double cover, $\iota: X \rightarrow X$ the involution, and assume given a $g_{5}^{1} X^{\prime} \rightarrow \mathbb{P}^{1}$ with simple ramifications. Then [1] in the induced map

$$
f^{(5)}: X_{5} \rightarrow X_{5}^{\prime}
$$

we have

$$
f^{(5)-1}\left(g_{5}^{1}\right)=C \cup \tilde{C}
$$

with $C$ and $\tilde{C}$ nonsingular and $\iota(C)=\tilde{C}$. Define a correspondence $D \subset C \times C$ by

$D=\left\{\left(x_{1}+\cdots+x_{5}, \iota x_{1}+\cdots+\iota x_{4}+x_{5}\right) \in C \times C: f^{(5)}\left(x_{1}+\cdots+x_{5}\right) \in g_{5}^{1}\right\}$

It is a fixed-point free nonsingular correspondence with $d=5$ of exponent $e=4$ (see [1]). Moreover $P=\operatorname{Prym}(X, \iota)$ so that $\operatorname{dim}(P)=g^{\prime}-1$ where $g^{\prime}=g\left(X^{\prime}\right)$. Therefore

$$
g(C)=g=d+e \operatorname{dim}(P)=4 g^{\prime}+1
$$

By a computation one finds

$$
|R|=8|r|=16\left(g^{\prime}+4\right)
$$


where $r$ is the ramification divisor of the $g_{5}^{1}$. Moreover

$$
2 g(D)-2=5(2 g-2)+16\left(g^{\prime}+4\right)=40 g^{\prime}+16\left(g^{\prime}+4\right)=56 g^{\prime}+64
$$

and it follows that

$$
g(D)=28 g^{\prime}+33
$$

and

$$
\bar{g}=14 g^{\prime}+17
$$

We have

$\operatorname{deg}\left(N_{\bar{D}}\right)=-g(D)+1+|R|=-28 g^{\prime}-33+1+16\left(g^{\prime}+4\right)=-12 g^{\prime}+32<0$

if $g^{\prime} \geq 3$ and

$$
h^{1}\left(N_{\bar{D}}\right)=-\chi\left(N_{\bar{D}}\right)=26 g^{\prime}-16
$$

Therefore we obtain

$h^{1}\left(T_{C_{2} \mid \bar{D}}\right)=h^{1}\left(N_{\bar{D}}\right)+h^{1}\left(T_{\bar{D}}\right)=\left(26 g^{\prime}-16\right)+\left(42 g^{\prime}+48\right)=68 g^{\prime}+32=6 \bar{g}-6-|R|$

which is compatible with the exact sequence (18).

3. Let $X$ be hyperelliptic of genus $\gamma \geq 3, f: \tilde{X} \rightarrow X$ an etale $3: 1$ morphism, and let $C=f^{(2)-1}\left(g_{2}^{1}\right)$ where $f^{(2)}: \tilde{X}_{2} \rightarrow X_{2}$. It can be shown that for general choice of $X$ and $f$ the curve $C$ is irreducible and nonsingular (see [5]). The induced map

$$
f^{(2)}: C \rightarrow \mathbb{P}^{1}=g_{2}^{1}
$$

is of degree 9 . If $x=y \in g_{2}^{1}$ then we have:

$$
f^{(2)-1}(x+y)=\left\{P_{i j}:=x_{i}+y_{j}: i, j=1,2,3\right\}
$$

where $\left\{x_{1}, x_{2}, x_{3}\right\}=f^{-1}(x)$ and $\left\{y_{1}, y_{2}, y_{3}\right\}=f^{-1}(y)$. Define a correspondence $D$ on $C$ by

$$
D\left(P_{12}\right)=P_{11}+P_{13}+P_{22}+P_{32}
$$

etc. Then one can show that this is a symmetric nonsingular and irreducible correspondence of degree $d=4$, exponent $e=3$, the corresponding PrymTyurin variety $\mathrm{P}$ is of dimension $\gamma-3$ and $g=g(C)=3 \gamma-5$. Moreover the 
number of moduli in this case can be computed to be $2 \gamma-1$ [5]. It is pretty clear that $R=0$ so that

$$
2 g(D)-2=4(2 g-2)=24 \gamma-48
$$

thus:

$$
\begin{gathered}
g(D)=12 \gamma-23 \\
\bar{g}=6 \gamma-11
\end{gathered}
$$

We have

$$
\operatorname{deg}\left(N_{\bar{D}}\right)=-g(D)+1=-12 \gamma+24<0
$$

so that

$$
h^{1}\left(N_{\bar{D}}\right)=-\chi\left(N_{\bar{D}}\right)=18 \gamma-36
$$

and we get

$$
h^{1}\left(T_{C_{2} \mid \bar{D}}\right)=h^{1}\left(N_{\bar{D}}\right)+3 \bar{g}-3=2(18 \gamma-36)
$$

which is compatible with the value given by the exact sequence (18).

\section{References}

[1] C. Birkenhake, H. Lange: Complex Abelian Varieties, second, augmented, edition, Springer Grundlehren n. 302 (2004).

[2] B. Fantechi: Deformations of symmetric products of curves, Contemporary Math. 162 (1994), 135-141.

[3] Green M., Lazarsfeld R.: A simple proof of Petri's theorem on canonical curves, Geometry Today, Roma 1984, Birkhauser 1985, 129-142.

[4] G. Kempf: Deformations of symmetric products, in Riemann surfaces and related topics - Proceedings of the 1978 Stony Brook Conference, 319-341, Princeton University Press 1980.

[5] H. Lange, H. Recillas: Polarizations of Prym varieties of pairs of coverings, Arch. Math. 86 (2006), 111-120.

[6] Lazarsfeld R.: A sampling of vector bundle techniques in the study of linear series, in Lectures on Riemann Surfaces, 500-559, World Scientific 1989. 
[7] E. Sernesi: Deformations of Algebraic Schemes, Springer Grundlehren b. 334 (2006). 